\title{
Large multi Gbit/s delays generated in an all-optical tunable delay line preserving wavelength and signal bandwidth
}

\author{
Sanghoon Chin and Luc Thévenaz \\ Ecole Polytechnique Fédérale de Lausanne, STI-NAM Station 11, CH-1015 Lausanne, Switzerland \\ sanghoon.chin@epfl.ch, luc.thevenaz@epfl.ch
}

\begin{abstract}
Large all-optical tunable delays are generated in a dispersive fiber by double wavelength conversion through cross gain modulation in semiconductor optical amplifiers. A $156 \mathrm{ps}$ pulse train is continuously delayed up to $14 \mathrm{~ns}$.

(C)2008 Optical Society of America

OCIS codes: (060.4370) Nonlinear optics, fibers; (060.2320) Fiber optic, amplifier and oscillators; (350.5500) Propagation
\end{abstract}

\section{Introduction}

The development of all-optically controlled delay lines and fast access memories is seen like one of the greatest challenge for photonic signal processing, since they are believed necessary for the development of the future all-optical packet routers in photonic communication systems. Over the last decade, successful experiments to widely control the group velocity of optical signal pulses have been reported that make use of optical resonance effects. Recently, a significant progress toward real applications has been developed since slow-light is experimentally and efficiently observed in optical fibers. These delaying techniques have the advantages of compatibility with fiber-optic communication systems, room-temperature operation at any wavelength and large data bandwidth. However, the maximum time delay that a pulse can experience in these schemes remains restricted to a few bits delay as a result of the considerable distortion due to the dispersion associated with the slow light process. The scientific challenge to produce a large amount of pulse delay with negligible distortion remains opened.

We here propose a new technique to improve to a large increment the pulse fractional delay, based on the combination of double wavelength conversion and propagation in a dispersive element [1-3]. The main progress in our configuration compared to previous works is to realize simply and efficiently the wavelength conversion using cross gain modulation in a semiconductor optical amplifier (SOA) in a compact and low power configuration. This type of conversion offers the key advantage to be efficient over a much broader wavelength range than parametric processes. This extends the range of delays obtained by the dispersive line and we could demonstrate experimentally a large range of optical delays with low distortion, up to tens of nanosecond for $156 \mathrm{ps}$ optical pulses.

\section{Principle}

Cross gain modulation (XGM) in a semiconductor optical amplifier (SOA) is usually described as a nonlinear interaction between two co- or counter-propagating beams, a strong pump wave at $v_{\text {pump }}$ and a weak probe wave at $v_{\text {probe }}$. Let suppose an intensity modulated pump light entering an SOA and a CW probe light simultaneously injected counter-directionally into the SOA. Due to gain modulation, the pump light will modulate the gain in the SOA [4]. In turn XGM in the amplifier will impose the pump modulation on the probe. Thus the intensity of the probe at the target wavelength is inversely modulated and carries the complementary data pattern, so essentially the same information as the pump modulation. Note that this process has the merit of high conversion efficiency and of no requirement for a phase matching condition. Besides, it can be polarization-independent if an SOA showing an polarization-independent gain is employed. After exiting the SOA, the probe light is launched into a highly dispersive optical medium such as long lengths of optical fiber [1,2] or even a highly dispersive grating [3]. The propagation velocity of the probe through the fiber can be continuously varied by simply tuning the probe wavelength as a result of the wavelength dependence of the group-velocity. The probe wave can thus exit the fiber with relative delays or advancements that can be estimated to first order as the product of probe wavelength change and the group-velocity dispersion (GVD) of the fiber. The delayed converted signal then experiences a second wavelength conversion in another SOA back to the original signal wavelength, restoring in the same process the original modulation pattern.

\section{Experiments and Results}

A $1571 \mathrm{~nm}$ distributed feedback (DFB) laser diode is used as signal source (pump) and its output is split using a 90/10 directional coupler. The light from the high power coupler branch is used to generate a data signal using an electro-optic modulator. In our setup shown in Fig. 1-(a) a $100 \mathrm{ps}$ FWHM pulse train at a $500 \mathrm{MHz}$ repetition rate is generated as test signal. Then the pulsed signal is boosted using an erbium-doped fiber amplifier 
(EDFA) before it is launched into the first SOA. A weak CW probe beam generated by tunable laser source (TLS) is also simultaneously launched into the first SOA in the direction opposite to the pulse direction to simply separate the input and the converted signals. Through the principle described above, XGM induces an intensity modulation on the probe, resulting in a wavelength converted signal that is inverted compared to the input pulses. The converted signal is then delivered to a dispersive medium that was simply in our test setup a $25 \mathrm{~km}$-long single mode fiber. After exiting from the fiber, the converted signal is amplified by another EDFA before entering the second SOA while the lower power channel from the DFB laser is injected into the converter. Using the same XGM process the second wavelength conversion is performed, so that the original signal pulse pattern is restored and the signal returns to its exact original wavelength.

The measured temporal delays of the output pulse as a function of the wavelength of the TLS are illustrated in Fig.1-(b). A great amount of time delay was obtained while the TLS wavelength was swept from $1526 \mathrm{~nm}$ to $1596 \mathrm{~nm}$. In our proposed method, we achieved a $\sim 14$ ns true time delay for $156 \mathrm{ps}$ FWHM optical pulse, resulting in a delay-to-pulse-width ratio of about 90 . This ratio can certainly be further improved using a more dispersive delay line and an even broader wavelength scanning range.

(a)

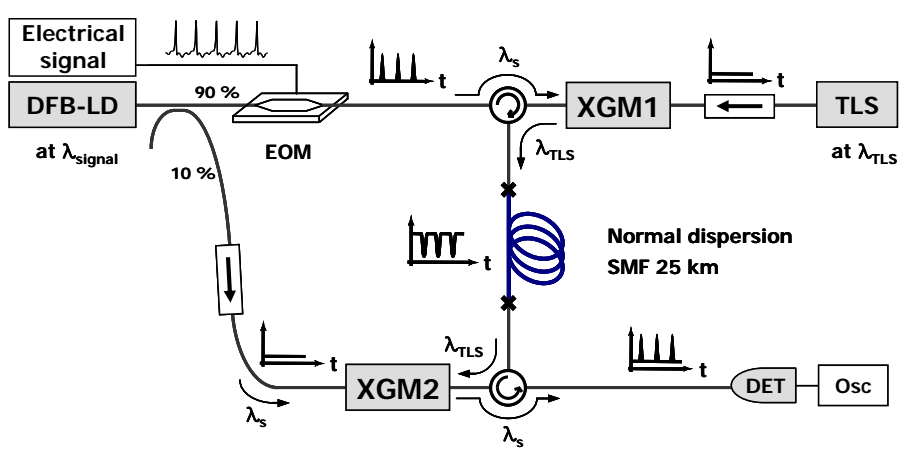

(b)

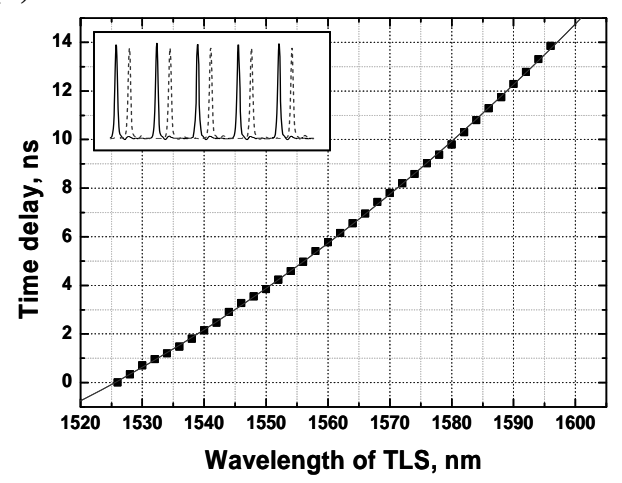

Fig.1: The experimental setup to realize the optical delay line is shown on the left and the relative pulse delays as a function of the TLS wavelength in the right. The insert shows the time delay experienced by the pulse train when the TLS wavelength is changed by 4 nm.

\section{Conclusions}

We have experimentally demonstrated the generation of large variable optical delays on a $6 \mathrm{Gbit} / \mathrm{s}$ data stream, based on the combination of GVD in optical fibers and double wavelength conversion using XGM in SOA. In this experiment, the maximum bit-rate of the data packet through the delay-line was restricted by the carrier recovery time of the used SOAs. However, it must be pointed out that our delay-line can be employed for high speed networks since error-free wavelength conversion at $160 \mathrm{Gbit} / \mathrm{s}$ has been realized [5]. The original signal wavelength is preserved and the signal bandwidth is only moderately modified by the chirp effects in the SOAs. We believe that this scheme can be a promising timing tool for future communication and microwave photonics systems.

\section{References}

[1] J. E. Sharping, Y. Okawachi and A.L. Gaeta, "Wide bandwidth slow light using a Raman fiber amplifier," Opt. Express 13, 6092-6098 (2005)

[2] Y. Okawachi, J. E. Sharping, C. Xu and A. L. Gaeta, "Large tunable optical delay via self-phase modulation and dispersion," Opt. Express, 14, 12022-12027 (2006).

[3] M. Fok, C. Shu, "Tunable Pulse Delay using Four-Wave Mixing in a 35-cm bismuth Oxide Highly Nonlinear Fiber and Dispersion in a Chirped Fiber Bragg Grating," in Proc. ECOC'06, Cannes, France (2006).

[4] T. Durhuus, B. Mikkelsen, C. Joergensen, S. L. Danielsen and K. E. Stubkjaer, "All-Optical Wavelength Conversion by Semiconductor Optical Amplifiers," J. Lightwave Technol., 14, 942-954 (1996)

[5] Y. Liu, E. Tangdiongga, Z. Li, S. Zhang, H. Waardt, G. D. Khoe and H. J. Dorren, "Error-Free All-Optical Wavelength Conversion at $160 \mathrm{~Gb} / \mathrm{s}$ Using a Semiconductor Optical Amplifier and an Optical Bandpass Filter," J. Lightwave. Technol., 24, 230-236 (2006) 